\title{
Flipping the doublesex switch with a piRNA
}

\author{
Cale Whitworth ${ }^{*}$ and Brian Oliver
}

\begin{abstract}
Recent work in the silkworm Bombyx mori has uncovered a novel Piwi-interacting RNA regulator of the sex determination switch doublesex.
\end{abstract}

\section{Connecting sex determination with sexual development}

Sex determination is one of the most important developmental decisions made by most organisms. This decision has major developmental consequences for the individual, as well as having enormous evolutionary impacts on the population. Sexual dimorphism is highly conserved, as egg-bearing females and sperm-bearing males are nearly universal, but the mechanisms of sex determination in animals are varied [1]. The best studied is the XX (female):XY (male) system found in mammals, Drosophila and many other species; however, there are other genotypic sex switches such as ZZ (male):ZW (female) found in birds, amphibians, reptiles and insects. In addition to genetic mechanisms of sex determination, there are known environmental and social sex determination switches, which have been identified in fishes and reptiles.

Despite considerable variation in primary sex determination signals, it is clear that there are ancient conserved factors that play roles in sexual development. One example is the doublesex- and mab-3-related transcription factor (DMRT) family of proteins that play important roles in sexual development in species including human, fish, birds, amphibians and insects [2]. Identifying primary sex determination switches and connecting these to conserved downstream factors, such as DMRTs, will be crucial in understanding the phenomenon of sex determination and sexual development. In this context, a recent study by Kiuchi and colleagues in Nature has identified the primary sex determination switch in the

\footnotetext{
* Correspondence: cale.whitworth@nih.gov

Section of Developmental Genomics, Laboratory of Cellular and Developmental Biology, National Institute of Diabetes and Digestive and Kidney Diseases, National Institutes of Health, 50 South Drive, Bethesda, MD 20892, USA
}

silkworm Bombyx mori and also linked its activity to a downstream DMRT ortholog required for sexual development [3].

\section{Identification of the female determinant in Bombyx mori}

The silkworm uses a ZZ (male):ZW (female) genotypic sex determination system. For 80 years, it has been known that the W chromosome in Bombyx mori carries a dominant female determinant, but identification of this factor has remained elusive. One issue contributing to the difficulty in this search is that the $\mathrm{W}$ chromosome is almost completely devoid of protein-coding genes and is composed primarily of retrotransposons [4]. Although the $\mathrm{W}$ is female determining, it shares many characteristics with a $\mathrm{Y}$ chromosome for many of the same evolutionary reasons, including lack of a homologous chromosome for recombination, which results in gene loss by means of 'Muller's ratchet'. One of the most significant findings by Kiuchi and colleagues is that a single Piwi-interacting RNA (piRNA), Feminizer (Fem), rather than a protein, is the sex-determining factor in the silkworm [3].

The definition of a female determinant, analogous to the male determinant on the $\mathrm{Y}$ in mammals, is that it must induce the female pathway. As in most insects, the downstream target or readout of this sex pathway is a doublesex gene $(B m d s x)$, which is spliced into female or male isoforms that match the genotypic sex of the individual. The male or female isoform regulates transcription of targets required to promote male or female sexual development, respectively [5,6]. By analyzing transcriptional profiles from male and female embryos around the time that sex-specific splicing of $B m d s x$ begins, Kiuchi et al. [3] discovered one sequence that was expressed specifically in female embryos and was located in the sex determining region of the $\mathrm{W}$ chromosome. This sequence was found to be a piRNA precursor that, when inhibited in female embryos, resulted in splicing of $B m d s x$ into the male isoform, effectively disrupting the female pathway in ZW females. Therefore, Fem alone is necessary for the 
regulated splicing of female $B m d s x$. But what is the target of the mature Fem piRNA complex?

As piRNAs have sequences matching their targets, the authors used complementarity searches to identify a novel gene, Masculinizer (Masc), located on the Z chromosome, possessing extensive similarity to the Fem sequence. Inhibition of Fem piRNA results in a corresponding increase in Masc mRNA levels, suggesting the existence of a piRNA-mediated regulatory relationship. Further strengthening the Fem-Masc relationship, the Masc coding region produces a piRNA with a perfect ping-pong signature match for Fem, indicating that these two can coordinate in a ping-pong amplification partnership to ensure efficient cleavage of Masc mRNA (Figure 1).

Masc is a zinc-finger protein with two main functions. First, Masc directly or indirectly regulates splicing of $B m d s x$ into the male isoform, as evidenced by RNA interference knockdown of Masc in embryos resulting in production of the female Bmdsx isoform. Second, Masc controls dosage compensation of Z-linked genes in $B$. mori as knockdown of Masc in embryos results in an upregulation of genes on the $\mathrm{Z}$ chromosome at early developmental stages that is accompanied by male-specific death before hatching. The coordinate regulation of sex and dosage compensation by Masc is strikingly similar to the role of Sex lethal $(S x l)$ in Drosophila melanogaster homogametic $(\mathrm{XX})$ females. As $S x l$ does not appear to play a role in $B$. mori sex determination $[7,8]$, it seems that Masc might functionally replace $S x l$ in the homogametic male silkworm. In this model, the W-linked piRNA Fem is the female determinant, whose main function is to inhibit Masc mRNA through piRNA-mediated cleavage (Figure 1a). In the absence of Fem (that is, ZZ males),
Masc regulates both $B m d s x$ splicing into the male isoform as well as dosage compensation (Figure 1b).

\section{Future directions}

Owing to experimental limitations, the authors were unable to demonstrate a phenotypic sex transformation through inhibition of Fem piRNA in females or of Masc piRNA or mRNA in males. Confirmation that manipulation of the Fem-Masc pathway results in sex transformations is an important next step in the validation of the Fem-Masc sex determination model in B. mori. Furthermore, it is known that the female determinant in $B$. mori can dominantly induce the female pathway. Therefore, demonstrating the sufficiency of the Fem piRNA to induce femaleness in $\mathrm{ZZ}$ males is another important additional line of evidence that will need to be included in future studies. Nevertheless, these important new findings will greatly facilitate such further work on a new model of sex determination in $B$. mori and perhaps in lepidopterans in general.

In addition to helping unravel an eight-decade-long mystery of the female determinant in B. mori, this study by Kiuchi and colleagues is the first to identify a piRNA acting as the primary sex determination signal in any species. Heterogametic sex chromosomes ( $\mathrm{Y}$ and $\mathrm{W}$ ) are repeat-rich and gene poor, which might facilitate the use of piRNAs and other small RNAs that can be maintained by tandem duplication. This work could have exciting implications for the role of piRNAs in other species, including those with ZZ:ZW systems, such as birds and amphibians, whose primary determinants have not been identified.

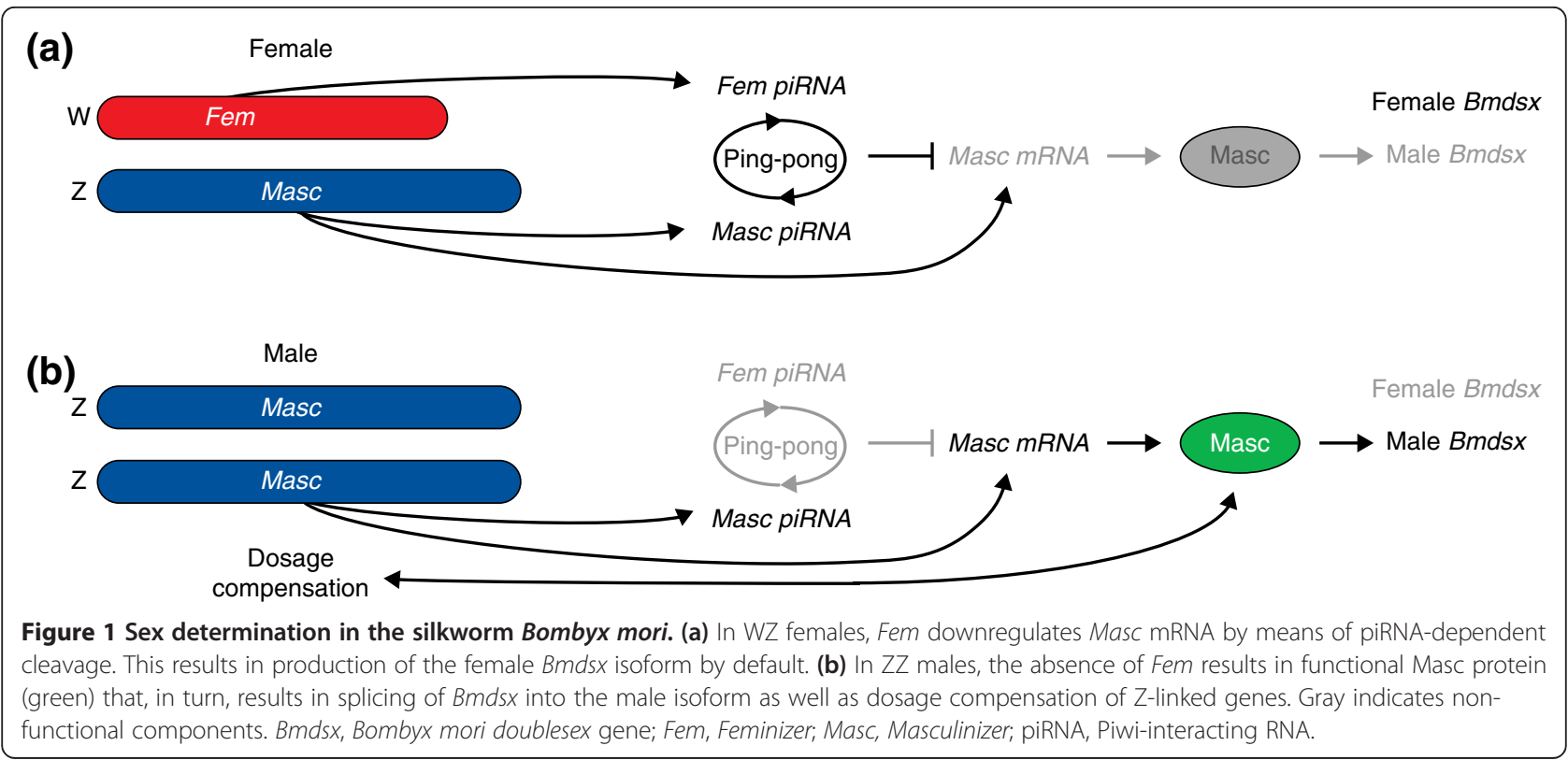




\section{Abbreviations}

Bmdsx: Bombyx mori doublesex gene; DMRT: Doublesex- and mab-3-related transcription factor; Fem: Feminizer; Masc: Masculinizer; piRNA: Piwi-interacting RNA; Sxl: Sex lethal.

\section{Competing interests}

The authors declare that they have no competing interests.

\section{Acknowledgements}

We thank our labmates for comments. This work was supported by the Intramural Research Program of the NIH, The National Institute of Diabetes and Digestive and Kidney Diseases (NIDDK).

Published: 24 June 2014

\section{References}

1. Gamble T, Zarkower D: Sex determination. Curr Biol 2012, 22:R257-R262.

2. Matson CK, Zarkower D: Sex and the singular DM domain: insights into sexual regulation, evolution and plasticity. Nat Rev Genet 2012, 13:163-174.

3. Kiuchi T, Koga H, Kawamoto M, Shoji K, Sakai H, Arai Y, Ishihara G, Kawaoka S, Sugano S, Shimada T, Suzuki Y, Suzuki MG, Katsuma S: A single femalespecific piRNA is the primary determiner of sex in the silkworm. Nature 2014, 509:633-636.

4. Abe H, Mita K, Yasukochi Y, Oshiki T, Shimada T: Retrotransposable elements on the W chromosome of the silkworm, Bombyx mori. Cytogenet Genome Res 2005, 110:144-151.

5. Ohbayashi F, Suzuki MG, Mita K, Okano K, Shimada T: A homologue of the Drosophila doublesex gene is transcribed into sex-specific mRNA isoforms in the silkworm, Bombyx mori. Comp Biochem Physiol B Biochem Mol Biol 2001, 128:145-158.

6. Fujii T, Shimada T: Sex determination in the silkworm, Bombyx mori: a female determinant on the $\mathrm{W}$ chromosome and the sex-determining gene cascade. Semin Cell Dev Biol 2007, 18:379-388.

7. Niimi T, Sahara K, Oshima H, Yasukochi Y, Ikeo K, Traut W: Molecular cloning and chromosomal localization of the Bombyx Sex-lethal gene. Genome 2006, 49:263-268.

8. Traut W, Niimi T, Ikeo K, Sahara K: Phylogeny of the sex-determining gene Sex-lethal in insects. Genome 2006, 49:254-262.

doi:10.1186/gb4181

Cite this article as: Whitworth and Oliver: Flipping the doublesex switch with a piRNA. Genome Biology 2014 15:118. 